\title{
Low-emittance radio-frequency electron gun using a gridded thermionic cathode
}

\author{
Takao Asaka, ${ }^{1,2,3, *}$ Takahiro Inagaki, ${ }^{1,2}$ Tamotsu Magome,,${ }^{1,2,3}$ Nobuyuki Nishimori, ${ }^{3}$ Yuji Otake, ${ }^{2}$ \\ Tsutomu Taniuchi, ${ }^{2}$ Kenich Yanagida, ${ }^{2}$ and Hitoshi Tanaka ${ }^{1,3}$ \\ ${ }^{1}$ RIKEN SPring-8 Center, 1-1-1 Kouto, Sayo, Hyogo 679-5148, Japan \\ ${ }^{2}$ Japan Synchrotron Radiation Research Institute (JASRI), 1-1-1 Kouto, Sayo, Hyogo 679-5198, Japan \\ ${ }^{3}$ National Institutes for Quantum and Radiological Science and Technology (QST), \\ 1-1-1 Kouto, Sayo, Hyogo 679-5148, Japan
}

(Received 26 March 2020; accepted 9 June 2020; published 23 June 2020)

\begin{abstract}
We developed an electron gun system capable of delivering high brightness beams to drive soft x-ray free-electron lasers (SXFEL). We modified the conventional radio-frequency (rf) gun concept by using a $50-\mathrm{kV}$ gun with a gridded thermionic cathode connected with a $238-\mathrm{MHz}$ acceleration cavity. We achieved a low target emittance by applying the following two methods: (1) to obtain a parallel beam after the control grid, we optimized the grid voltage to compensate for the electric potential distortion near the grid (i.e., the lens effect); and (2) to avoid emittance growth due to the space charge effect at low energy regions, the $50-\mathrm{kV}$ gun was connected to a $238-\mathrm{MHz}$ rf cavity with a minimum distance, which immediately accelerated the beam energy to $500 \mathrm{keV}$. We built a gun test stand to verify the high beam performance, i.e., a normalized emittance of less than $4 \mathrm{~mm} \operatorname{mrad}$ with a bunch charge of $1 \mathrm{nC}$ and a pulse length shorter than $0.7 \mathrm{~ns}$, as predicted by CST and PARMELA codes. Our proof-of-performance experiments demonstrated that a projection emittance of $1.7 \mathrm{~mm}$ mrad was obtained as a core part, representing $60 \%$ of all extracted electrons and the measured emittance agreed well with the simulation value of $2.0 \mathrm{~mm}$ mrad. This paper presents an overview of the electron gun system and our proof-ofperformance experimental results.
\end{abstract}

DOI: 10.1103/PhysRevAccelBeams.23.063401

\section{INTRODUCTION}

$\mathrm{X}$-ray and soft X-ray free-electron lasers (XFEL [1-5] and SXFEL [6-8]) have long served as powerful tools for exploring innovative science over a wide variety of academic fields, including chemistry, materials science, biology, nonlinear optics, and many other domains. One of the key requirements for an FEL accelerator system is to provide a low-emittance and high-peak current electron beam to obtain a sufficient amplification gain, enabling FEL power saturation with a reasonably short undulator length. The low-emittance electron sources developed for XFELs fall into two principal categories: photocathode radio-frequency (rf) guns [9-12] and high voltage thermionic guns [13]. The photocathode rf guns have been used in many facilities as they provide a smaller and homogeneous emittance and have a more compact size. The photocathode however requires a complex drive laser system at ultraviolet

\footnotetext{
*asaka@ spring8.or.jp
}

Published by the American Physical Society under the terms of the Creative Commons Attribution 4.0 International license. Further distribution of this work must maintain attribution to the author(s) and the published article's title, journal citation, and DOI. wavelength, which demands laser specialists to maintain stable and reliable operations of the rf guns. The high voltage thermionic gun is nearly maintenance free, but it requires a large oil-filled high voltage pulse modulator to generate a microsecond $500 \mathrm{keV}$ beam. In addition, an electromagnetic chopper system needs to be installed downstream of the gun not only to cut a short pulse of $1 \mathrm{~ns}$ from the microsecond beam, but also to preserve the low electron beam emittance [14].

As a third type of FEL electron source, we developed an rf gun using a gridded thermionic cathode, which provides a sufficiently small beam emittance with pulse length shorter than $0.7 \mathrm{~ns}$ and offers the prime advantage of the thermionic gun (ease of maintenance) while not requiring the complex high voltage pulse modulator and chopper systems. Our system features a $50-\mathrm{kV}$ thermionic gun connected to a 238-MHz rf cavity in order to immediately increase the beam energy to $500 \mathrm{keV}$ or even higher. This system uses a commercially available gridded cathode that provides an electron pulse shorter than $1 \mathrm{~ns}$, which assures high capture efficiency for the subsequent rf acceleration cavity. The electron bunch from the present electron source will be compressed by a velocity bunching system consisting of $476-\mathrm{MHz}$ and S-band rf cavities and two magnetic bunch compressors, similarly to SACLA [14]. 
The bunched beam will be accelerated to $3 \mathrm{GeV}$ by a C-band acceleration system [15]. Our simulation study shows generation of $3 \mathrm{GeV}$ electron beam with peak current greater than $2 \mathrm{kA}$ and a normalized slice emittance below $2 \mathrm{~mm}$ mrad is feasible with our $3 \mathrm{GeV}$ linear accelerator system design. This satisfies the SXFEL requirement.

An initial concern about our schema was how to suppress the emittance growth that resulted from the distorted electric potential (lens effect) near the grid mesh next to the cathode [16]. The lens effect originates from a mismatch between grid and gun high voltages, which forms the unnecessary electric field to transversally kick the electrons passing the grid $[17,18]$. The lens effect is considered to be controllable by adjusting the grid voltage to compensate the distortion of the electric potential given by a gun high voltage of $50 \mathrm{kV}$ [19]. To confirm this approach, we used computer simulations to determine the optimum conditions for making the grid transparent for the extracted beam in terms of the transverse perturbation around the grid and achieving a small emittance value. Based on the simulation results, we designed an electron gun system with a gridded thermionic cathode and built a gun test stand to verify the beam performance.

This paper provides an overview of our electron gun system with the gridded thermionic cathode, the system design details and our proof-of-performance experiments. Section II outlines the design strategy and basic design for our electron gun system. Next, Sec. III describes in detail the methods and mechanical designs we used to achieve the target low emittance. Finally, Sec. IV summarizes the beam performance measurements at the gun test stand and compares our experimental results with particle tracking simulations.

\section{OVERVIEW AND DESIGN STRATEGY FOR THE rf GUN SYSTEM}

In this section, we review our gun system and design strategy to support XFEL applications with a simple and compact rf gun using a gridded thermionic cathode. The system comprises a $50-\mathrm{kV}$ gun with a gridded thermionic cathode, a magnetic lens, a 238-MHz rf cavity, and a beam collimator. The gun generates low-energy, short-pulsed, and homogeneous cylindrical electron beams with an initial normalized emittance of about $1 \mathrm{~mm}$ mrad with optimized grid voltage. Then, an axially symmetric magnetic lens focuses the beam so as not to spread it widely over the minimized distance to the downstream rf cavity. The 238$\mathrm{MHz}$ rf cavity immediately accelerates the extracted beam to $500 \mathrm{keV}$ or higher to suppress emittance growth. The collimator just after the cavity adjusts the bunch charge depending on the purpose of injecting electron beams into a ring or driving FELs.

To generate a pulsed beam without a short-pulse laser system used in photocathode rf guns, we selected a commercially available gridded thermionic cathode, EIMAC
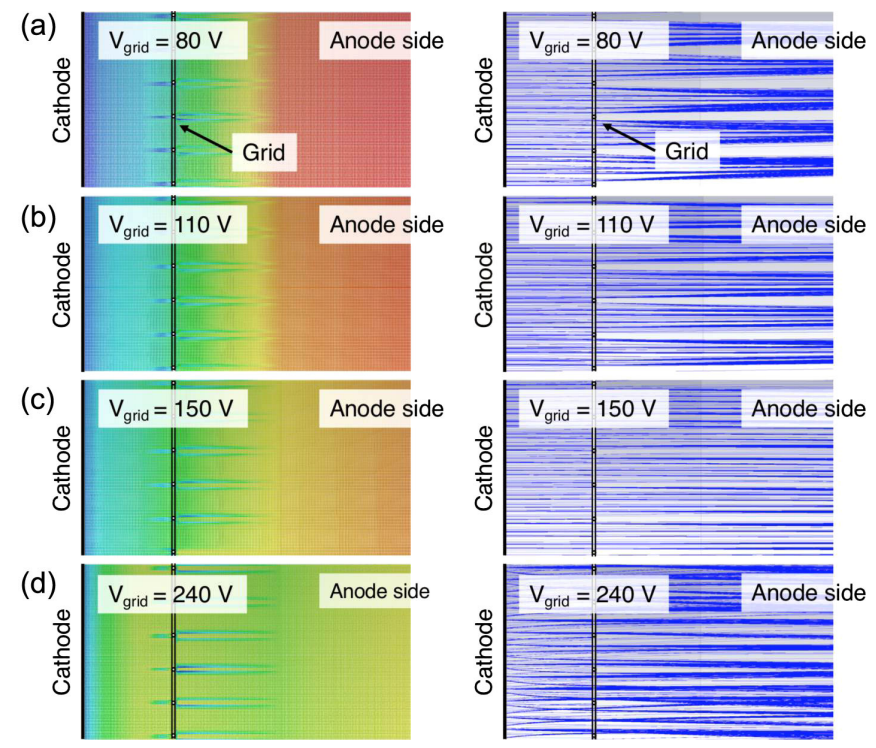

FIG. 1. Electric field distributions (left) and beam trajectories (blue lines) near the grid of Y-845 cathode (right) obtained with CST simulations. The symbols $V_{\text {grid }}$ represent the grid voltage and symbols (a), (b), (c), and (d) correspond to grid voltage conditions of $80,110,150$, and $240 \mathrm{~V}$, respectively. The color map shows the electric field strength (from blue at $0 \mathrm{~V} / \mathrm{m}$ to red at $1 \mathrm{MV} / \mathrm{m})$.

Y845 produced by CPI Inc. [20]. The gun high voltage was configured to be $50 \mathrm{kV}$, a relatively low voltage that confers the following three advantages: (1) it relaxes voltage endurance conditions, enabling reliable operation of the high voltage charger in the air without requiring insulation oil; (2) it simplifies the driving power supply by allowing use of solid-state power devices and consequently making the power-supply compact; and (3) it reduces the grid voltage to a few hundred volts, which can be provided by such a commercially available pulser as a fast pulse generator made by Kentech Instruments Ltd.

The normalized thermal emittance of the Y-845 cathode with radius of $4 \mathrm{~mm}$ is estimated to be $0.93 \mathrm{~mm}$ mrad at the cathode temperature of $1270 \mathrm{~K}$ for Maxwell-Boltzmann distribution. To preserve the initial small emittance, we optimized the mechanical and electrical parameters of the gun main components (such as the cathode, grid mesh and anode) so that the electric potential around the grid has a flat equipotential surface over the grid mesh as shown in Fig. 1. This is an equivalent condition with the grid removed. The diagrams show the simulated electric field distributions around the grid and electron beam trajectories for various grid voltages. In the simulation, the gun high voltage is fixed at $50 \mathrm{kV}$, while the grid voltage against the cathode is changed from 80 to $240 \mathrm{~V}$. As the grid voltage increases from $80 \mathrm{~V}$, the focusing electric field at the grid entrance decreases (see Fig. 1, left) and the lens effect almost disappears at a grid voltage of $150 \mathrm{~V}$, resulting in parallel beam trajectories after the grid (see Fig. 1, right). To simplify 


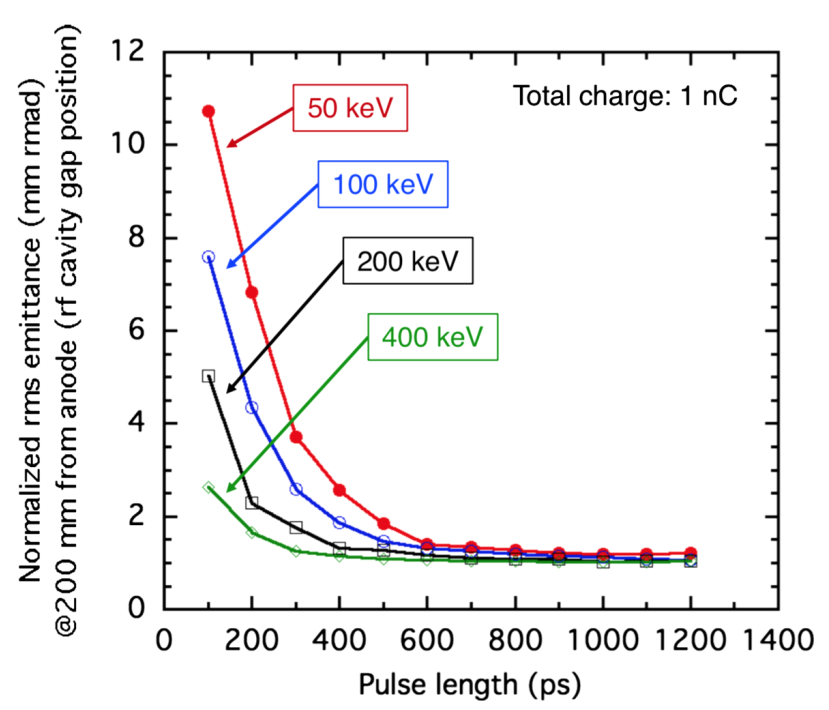

FIG. 2. Dependence of emittance growth on the beam pulse length for various gun high voltages. The extraction charge was fixed at $1 \mathrm{nC}$.

the optimization process, we also fixed the extraction charge at $1 \mathrm{nC}$, which is high enough for beam injection to a storage ring and an FEL. Since this grid-voltage optimization process limits flexibility to change the emission charge from the cathode, a round-shaped collimator was installed after the rf acceleration cavity to control the bunch charge so as not to seriously degrade beam emittance.

Beam transport from the gun anode exit to the cavity entrance at the low energy of $50 \mathrm{keV}$ potentially causes serious emittance growth due to the space charge force arising from static electric potential inside the electron beam itself. A shorter transport distance can alleviate the emittance growth due to the nonlinear space charge force which cannot be compensated by an emittance compensation solenoid [21]. We therefore embedded a single magnetic lens at the cavity entrance, making the distance between the anode exit and the center of the acceleration gap as short as possible. Instead of a peak current, we used a pulse length as the optimization parameter because the optimal peak current is equivalent to the optimal pulse length under a constant extraction charge. We thus investigated the dependence of emittance growth on beam pulse length. Figure 2 shows the simulation results. It is clear that a longer pulse length allows a lower gun high voltage while maintaining small emittance growth. At a gun high voltage of $50 \mathrm{kV}$, the shortest pulse length that does not significantly increase the emittance is found to be about $600 \mathrm{ps,}$ which is an ideal pulse length of the grid pulse voltage.

We selected an rf frequency of $238 \mathrm{MHz}$ to capture the short-pulsed beam of $600 \mathrm{ps}$, suppressing the energy spread caused by the curvature of an rf voltage within a level of around ten percent.

\section{DESIGN DETAILS AND EXPECTED PERFORMANCE}

\section{A. 50-kV gridded thermionic gun}

We used CST simulations to design mechanical shape and arrangement of the $50-\mathrm{kV}$ gun components such as cathode, Wehnelt and anode electrodes. The simulations optimize the focusing electric field which compensates for the defocusing by the electron beam space charge effect, enabling us to suppress the emittance growth from the initial thermal emittance and to maintain homogeneous beam distribution. The CST simulation takes into account the initial thermal emittance.

Figure 3 shows the beam aperture, arrangement, and mechanical shape for each component of the gun system,

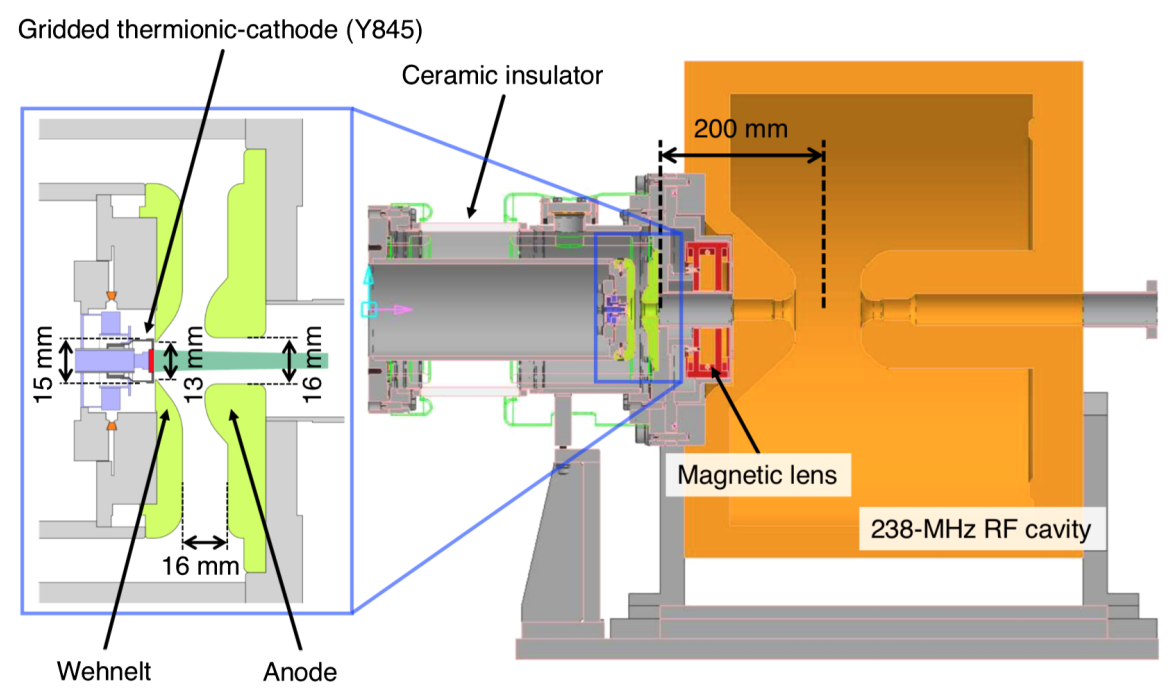

FIG. 3. Beam aperture, arrangement, and mechanical shape of each component along the rf gun system. The green belt in the inset represents the electron beam envelope simulated by CST code. 


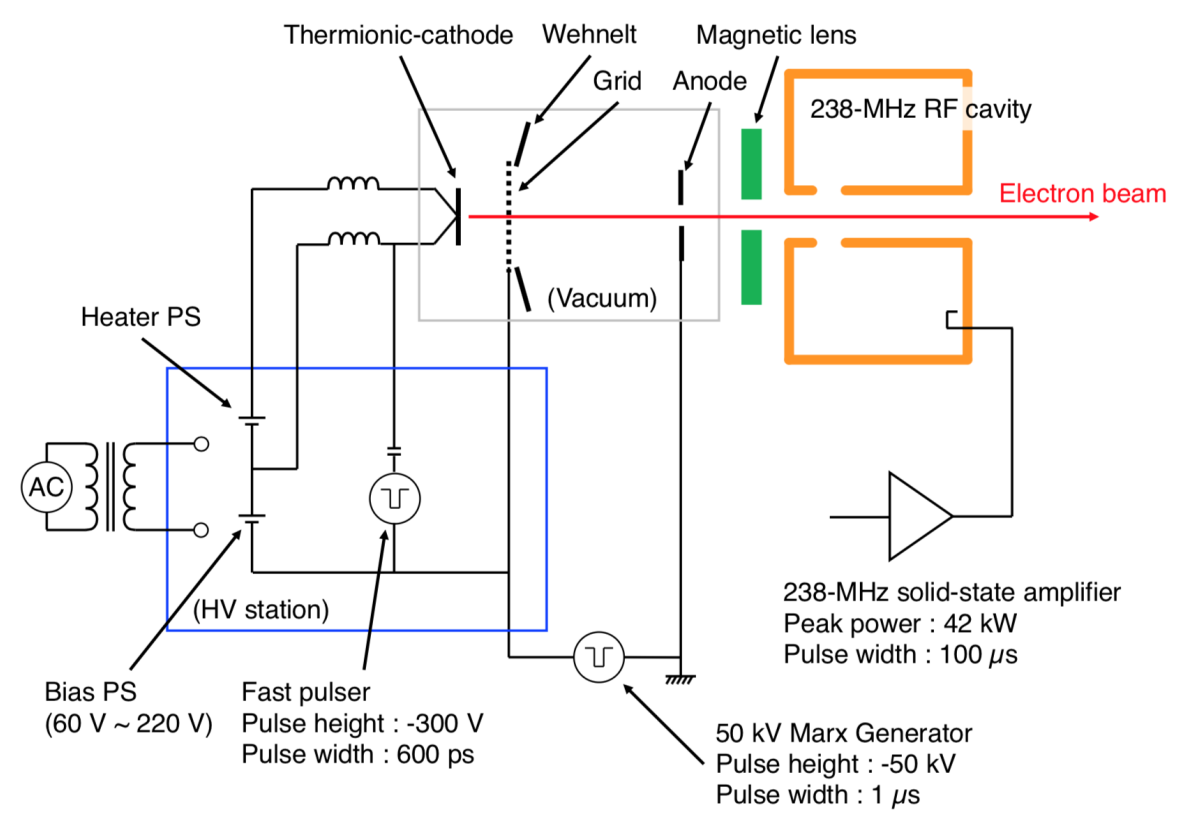

FIG. 4. Circuit diagram of the rf gun using thermionic gridded cathode.

together with the electron trajectories emitted from the cathode to the gun exit. The Y-845 cathode is composed of a cathode electrode and a grid mesh attached to the cathode with a gap of $0.14 \mathrm{~mm}$. The diameters of the cathode and grid are 8 and $15 \mathrm{~mm}$, respectively. This gridded cathode is located $0.5 \mathrm{~mm}$ upstream from the entry of the Wehnelt electrode with a beam aperture of $13 \mathrm{~mm}$ in diameter. This aperture is smaller than that of the grid, to slightly focus the beam as it exits the grid. The transverse beam size decreases smoothly as it moves downstream, due to a focusing electric field given by the Wehnelt with an opening angle of 52.5 degrees. A parallel cylindrical beam with a diameter of $6 \mathrm{~mm}$ is obtained at the anode exit. The physical aperture of the anode electrode of $16 \mathrm{~mm}$ in diameter and its distance from the cathode surface are optimized to achieve a flat equipotential surface of the electric field over the transverse beam size. This optimization also results in a low electric-field strength of less than $10 \mathrm{MV} / \mathrm{m}$ at the anode surface, which is sufficiently lower than the limit of electric discharge when $-50 \mathrm{kV}$ is applied to the cathode.

Figure 4 illustrates the circuit diagram of the present $\mathrm{rf}$ gun system. A $50 \mathrm{kV}$ Marx generator is employed to stably and reliably apply $-50 \mathrm{kV}$ on the cathode, grid, and Wehnelt electrodes with a pulse duration of $1 \mu s$ [22]. The generator is composed of six SiC MOSFETs [23], each of which has a withstanding voltage of $13 \mathrm{kV}$. To prevent dark current emissions from the cathode, the grid and Wehnelt voltages should be lower than $-40 \mathrm{~V}$ against the cathode voltage. A commercially available fast pulser (e.g., a product by Kentech Instruments Ltd.) which generates a peak pulse voltage of $300 \mathrm{~V}$ with a pulse length of $0.6 \mathrm{~ns}$ is applied on the cathode as a gate switch for beam generation. A variable dc bias voltage is also applied on the cathode to control the cathode-grid voltage.

Table I summarizes the main beam parameters at the gun exit obtained with CST simulation. A normalized emittance of $1.3 \mathrm{~mm}$ mrad at a bunch charge of $1 \mathrm{nC}$ is predicted. The phase space distributions at the gun exit obtained with CST simulation are used as inputs for PARMELA simulation which is performed from the gun exit to a diagnostic system downstream the $238-\mathrm{MHz}$ cavity.

\section{B. Magnetic lens}

To focus the extracted beam from the gun, a magnetic lens is adopted instead of a solenoid lens. Reduction of leakage field from the magnetic lens along the beam propagating direction is critically important to minimize the distance from the gun exit to the rf acceleration gap as well as to maintain sufficient field separation among three components of the present rf gun system (the $50 \mathrm{kV}$ gun, the magnetic lens, and the rf cavity). For this purpose, we developed an axially symmetric magnetic lens shown in Fig. 5(a) where the main solenoid coil has correction coils and soft iron yokes at both ends to reduce field leakage. The solenoid field is confined in a longitudinal length of $150 \mathrm{~mm}$ with this design. Outside of this area, the leakage field is lower than the earth's magnetic field. To embed the

TABLE I. Main beam parameters at the $50-\mathrm{kV}$ thermionic-gun exit obtained with CST simulation.

\begin{tabular}{lc}
\hline \hline Charge (current/pulse width) & $1 \mathrm{nC}(1.7 \mathrm{~A} / 0.6 \mathrm{~ns})$ \\
Energy & $50 \mathrm{keV}$ \\
Normalized emittance & $1.3 \mathrm{~mm} \mathrm{mrad}$ \\
\hline \hline
\end{tabular}


(a)

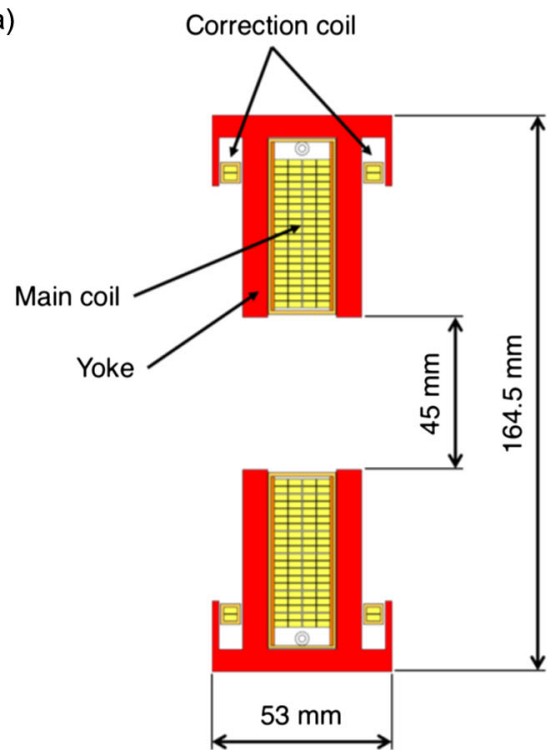

(b)

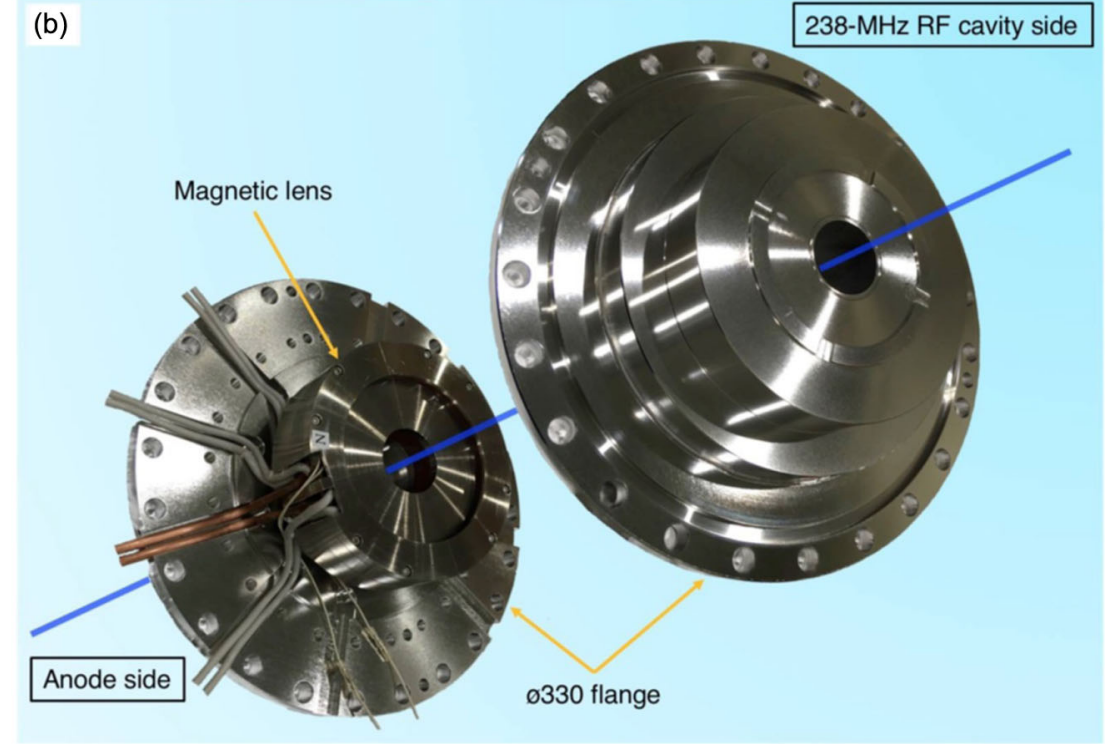

FIG. 5. (a) Cross section of the magnetic lens with the correction coils and (b) metal casing enclosing the magnetic lens.

magnetic lens in the cavity, the magnetic lens is enclosed in a metal casing with two vacuum flanges. This built-in magnetic lens provides a vacuum beam pipe as shown in Fig. 5(b). The distance from the gun exit to the center of the acceleration gap is shortened to $200 \mathrm{~mm}$, as shown in Fig. 3. The newly developed built-in magnetic lens together with the minimized distance from the gun exit to the acceleration gap allows the beam to be transversely focused to $6 \mathrm{~mm}$ in diameter at the center of the acceleration gap with a charge of $1 \mathrm{nC}$ and a pulse length of $0.6 \mathrm{~ns}$.

\section{The 238-MHz rf acceleration system}

A 238-MHz rf cavity needs to satisfy the following conditions: (1) a high field gradient to accelerate a $50 \mathrm{keV}$ beam up to $500 \mathrm{keV}$ which is equivalent to a shunt impedance of $6 \mathrm{M} \Omega$ for $34 \mathrm{~kW}$ input power; (2) a structure minimizing the beam transport distance from the anode exit to the rf cavity acceleration gap; and (3) an axially symmetric acceleration field distribution.

The required high shunt impedance can be satisfied with a cavity structure using an acceleration gap with a narrow beam aperture. This is because the structure can provide a high and relatively uniform acceleration field in the beam aperture without increase of manufacturing costs. The only disadvantage is the narrow beam aperture which can be overcome by keeping the beam size small enough through the cavity. By using PARMELA simulation, the aperture of the cavity was finally determined to be $22 \mathrm{~mm}$ in diameter, which is about 3 times larger than the beam size of $6 \mathrm{~mm}$ in diameter described in Sec. III B, providing the relaxed alignment tolerance.

The acceleration gap is placed upstream of the $238-\mathrm{MHz}$ cavity to minimize the beam transport distance, as shown in Fig. 3. In addition, the cavity is designed to maintain electromagnetic field symmetry in the beam propagating region while symmetry breaking components such as an $\mathrm{rf}$ input coupler and frequency tuners need to be installed. We installed an input coupler and tuners at the downstream sidewall where the surface current density is low and the acceleration gap is not directly seen.

The 238-MHz rf cavity is driven by a solid-state amplifier with a peak power of $40 \mathrm{~kW}$ and a pulse duration of $100 \mu \mathrm{s}$, as shown in Fig. 4. This amplifier is composed of 36 power (laterally-diffused metal-oxide semiconductor) FETs, each of which provides rf power of $1.5 \mathrm{~kW}$. All the rf pulses from the 36 semiconductors are superimposed using a 36-way cavity-type combiner. The superimposed rf power is fed into the cavity through a 39D-type coaxial transmission line. This solid-state amplifier is compact in size and reliable in operations with low rf noise. Table II summarizes the main parameters of the 238-MHz rf cavity system.

\section{Expected system performance}

We evaluated the system performance from the $50-\mathrm{kV}$ gun exit to a diagnostic system downstream of the

TABLE II. The main rf parameters of the 238-MHz rf cavity system.

\begin{tabular}{lcc}
\hline \hline Parameter & Design & Achieved \\
\hline Resonant frequency & $238 \mathrm{MHz}$ & $238 \mathrm{MHz} \pm 100 \mathrm{kHz}$ \\
$Q_{0}$ & 25400 & 23000 \\
Shunt impedance & $>6 \mathrm{M} \Omega$ & $6.17 \mathrm{M} \Omega$ \\
rf input power & $42 \mathrm{~kW}$ & $42 \mathrm{~kW}$ \\
rf pulse width & $100 \mu \mathrm{s}$ & $100 \mu \mathrm{s}$ \\
Repetition rate & $1-30 \mathrm{~Hz}$ & $1-30 \mathrm{~Hz}$ \\
\hline \hline
\end{tabular}




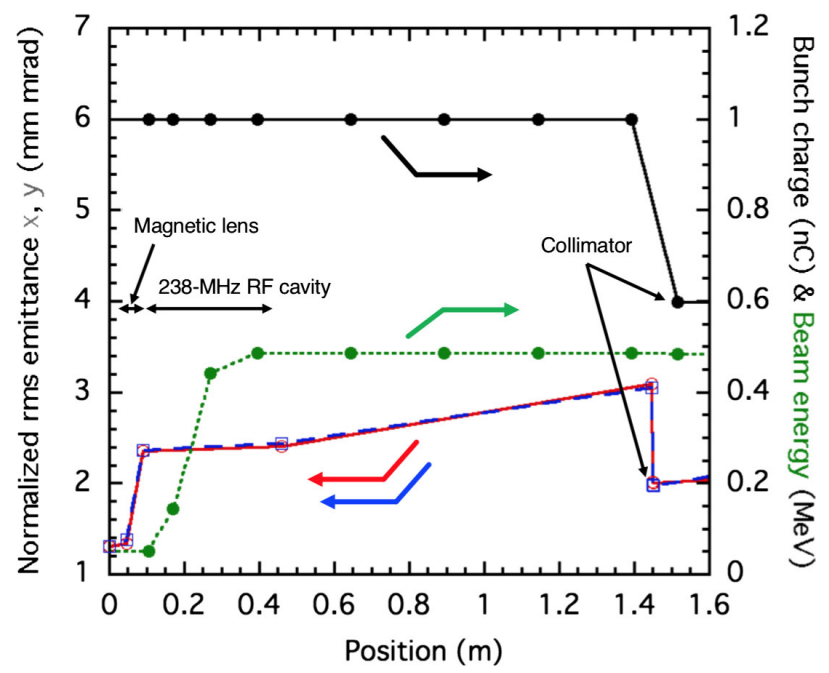

FIG. 6. The normalized emittance, bunch charge, and beam energy along the rf gun system obtained with PARMELA simulation. The origin of the horizontal axis represents the exit of the $50-\mathrm{kV}$ thermionic gun. The red solid and blue dashed lines represent evolutions of the horizontal and vertical projected normalized emittance (left axis), respectively. The black solid and green dotted lines represent evolutions of the bunch charge and beam energy (right axis), respectively.

238-MHz rf cavity with PARMELA simulation. Figure 6 shows the simulation results with the space charge effect included. The solenoid field by the magnetic lens is adjusted so that the normalized emittance at the $238 \mathrm{MHz}$ rf cavity is minimized without beam loss. The normalized emittance in the magnetic lens increases up to $2.5 \mathrm{~mm}$ mrad with a bunch charge of $1 \mathrm{nC}$ due to the space charge effect. The beam collimator is installed $1-\mathrm{m}$ downstream from the $238-\mathrm{MHz}$ rf cavity exit. The normalized emittance at this position increases to $3.1 \mathrm{~mm} \mathrm{mrad}$. The beam collimator can scrape the outer part of the beam to provide

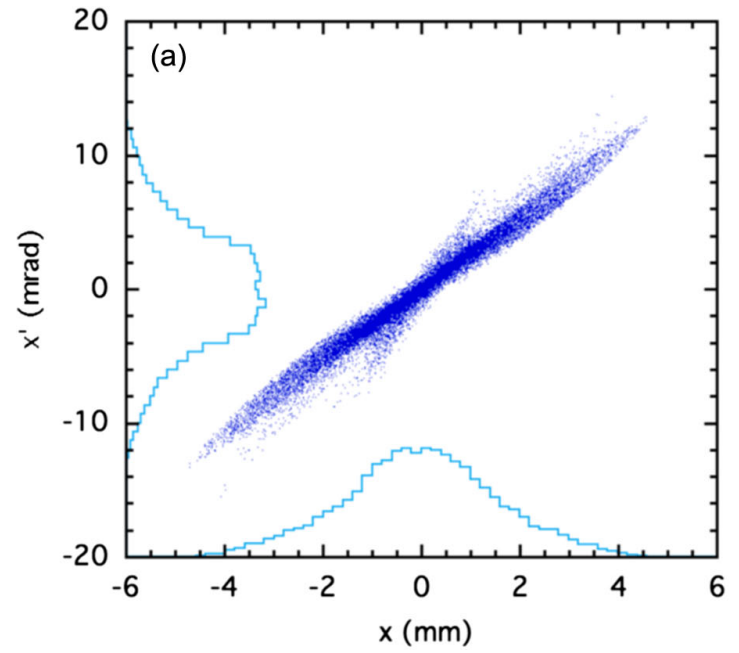

smaller emittance depending on the application. The simulation demonstrates that the $60 \%$ core part of the extracted beam has a normalized emittance of $2.0 \mathrm{~mm}$ mrad with a bunch charge of $0.6 \mathrm{nC}$. This indicates emittance growth from the initial thermal emittance of $0.93 \mathrm{~mm} \mathrm{mrad}$ is small in the present gun system. The particle distributions in the horizontal phase space and the longitudinal phase space after the $238 \mathrm{MHz}$ rf cavity exit are shown in Fig. 7.

\section{PROOF-OF-PERFORMANCE EXPERIMENTS}

\section{A. The gun test stand for proof of performance}

We built a gun test stand to demonstrate the beam performance of our rf gun. Figure 8 illustrates the gun test stand composed of a $50-\mathrm{kV}$ electron gun, a $238-\mathrm{MHz}$ rf cavity, two additional magnetic lenses, and a beam diagnostic system to measure the bunch charge, the beam energy, the beam profile, and the projected transverse beam emittance at the exit of the rf gun system. Figure 9 shows a photo of the gun test stand. A $200 \mu \mathrm{m}$-thick YAG:Ce screen is used to measure the beam profile in high spatial resolution. A random shutter CCD camera with a telecentric objective lens is used to measure the screen image over a size of $\pm 30 \mathrm{~mm}$ at a high spatial resolution of $31 \mu \mathrm{m}$ without distortion.

\section{B. Measured beam performance}

The bunch charge was measured using a Faraday cup with a digital oscilloscope and set to be $1 \mathrm{nC}$ for the following measurements. The beam energy at the gun exit was derived from a time of flight (TOF) measurement between two wall current monitors. The energy resolution of the TOF method is estimated to be $\pm 2 \%$ for $500 \mathrm{keV}$ electron beam. Red crosses in Fig. 10 show measured beam energies as a function of the rf power fed into the $238-\mathrm{MHz}$

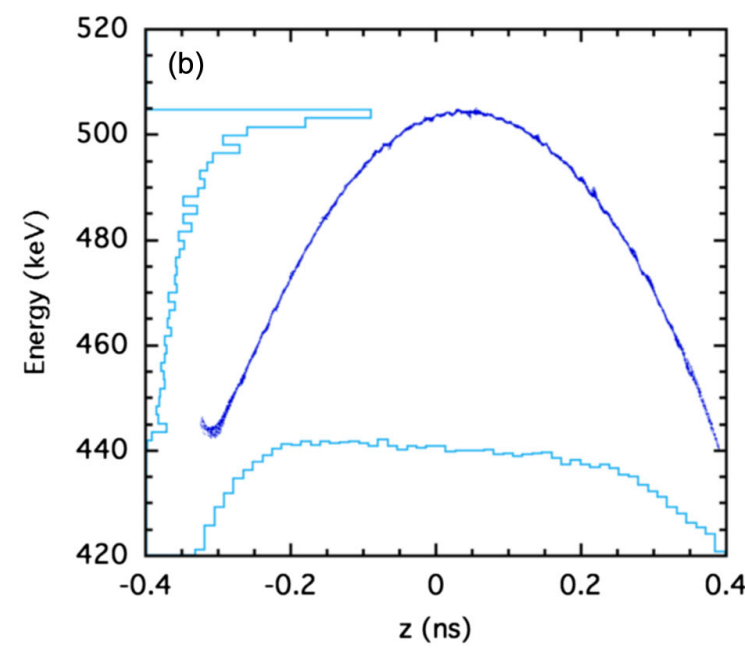

FIG. 7. Particle distributions in horizontal (a) and longitudinal (b) phase spaces at the 238-MHz rf cavity exit obtained with PARMELA simulation. 


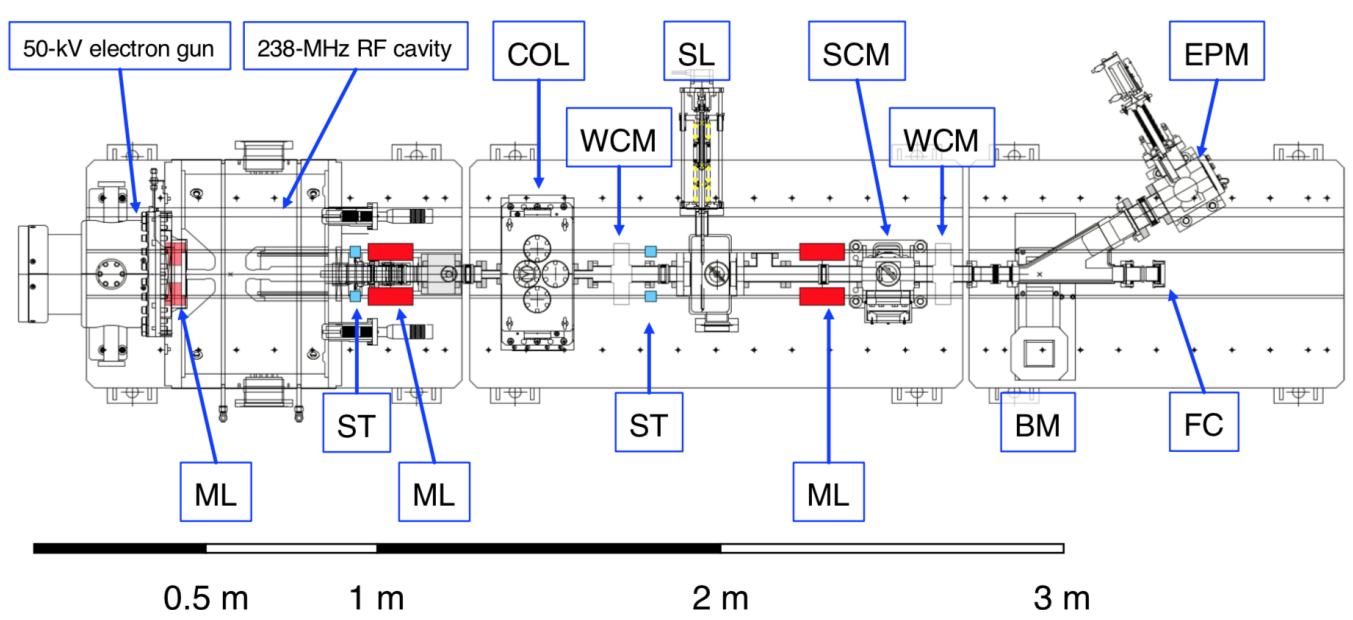

FIG. 8. Layout of the gun test stand. The symbols ML, ST, COL, SL, WCM, FC, SCM, EPM, and BM represent a magnetic lens, a steering coil, a beam collimator, a beam slit, a wall current monitor, a Faraday cup, a screen monitor, an energy profile monitor, and a $30^{\circ}$ bending magnet, respectively.

rf cavity. The measurement agrees well with a calculation given by the blue solid line using the measured shunt impedance of $6.17 \mathrm{M} \Omega$.

The energy profile was measured with a beam profile monitor located in the energy dispersive part downstream of the bending magnet with a deflection angle of $30^{\circ}$. The linear dispersion is estimated to be $0.22 \mathrm{~m}$ at the profile monitor position, providing a horizontal position sensitivity of $0.22 \mathrm{~mm}$ against a relative energy deviation of $0.1 \%$. The measured beam profile shown by the red line in Fig. 11(a) is formed by the energy dispersion effect and the beam size determined by the beta function and emittance. The measured profile agrees well with PARMELA simulation given by the blue line. The energy profile obtained from the simulation is shown in Fig. 11(b). The energy spread is evaluated to be $4.2 \%$ in the $60 \%$ core part at the $500 \mathrm{keV}$ beam with a bunch charge of $1 \mathrm{nC}$. The long tail in the

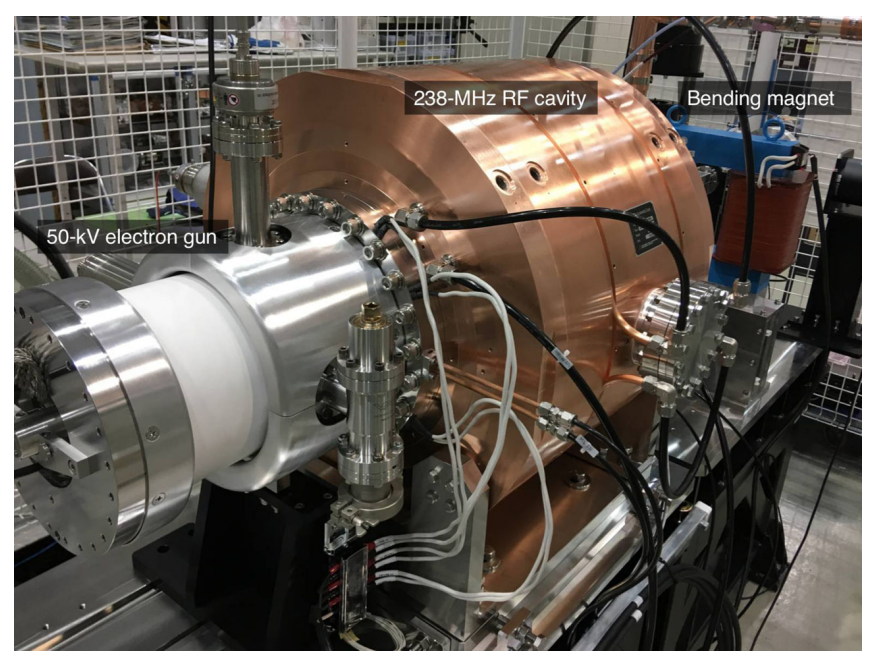

FIG. 9. Picture of the gun test stand. low-energy side is caused by the rf curvature of the 238$\mathrm{MHz}$ cavity for input beam pulse length of $0.6 \mathrm{~ns}$.

The projected horizontal emittance was measured by the slit scan method at a beam energy of $500 \mathrm{keV}$ and a bunch charge of $1 \mathrm{nC}$. The beam slit produces a sheetshaped beamlet from a round beam. A slit width of $100 \mu \mathrm{m}$ provides a charge low enough to suppress the beam divergence due to the space charge effect to a negligibly small level. This condition ensures that the beam emittance is dominant in horizontal widening of the beamlet cut out. By scanning the beam slit over the beam profile, the intensity and angular spread of the beamlet in the horizontal phase space were measured. Figure 12(a) shows the

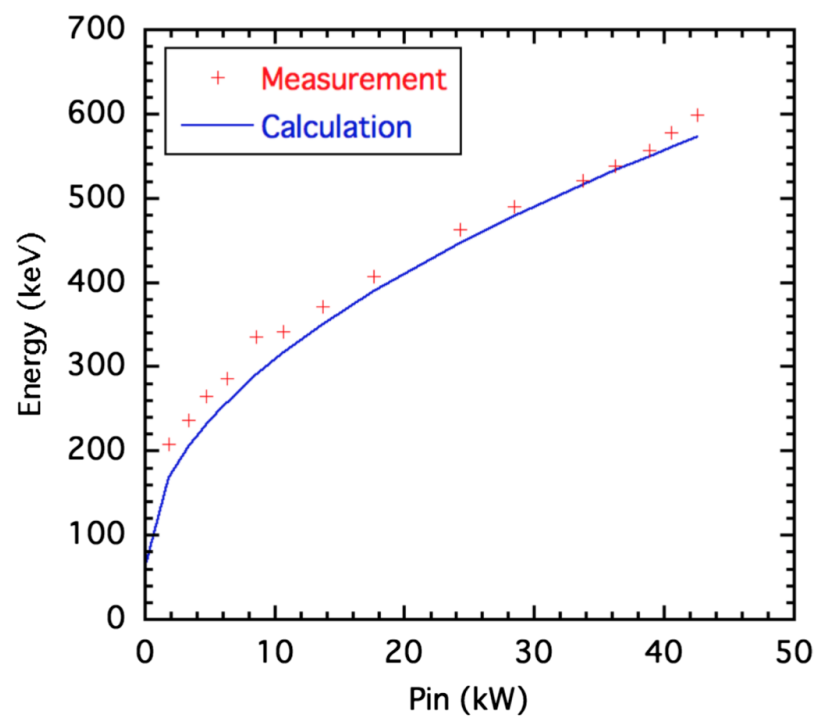

FIG. 10. Beam energy dependence on the rf input power to the 238-MHz rf cavity. The plus signs and solid line represent the measured data and calculation with a shunt impedance of 6.17 M $\Omega$ for the 238-MHz rf cavity, respectively. 

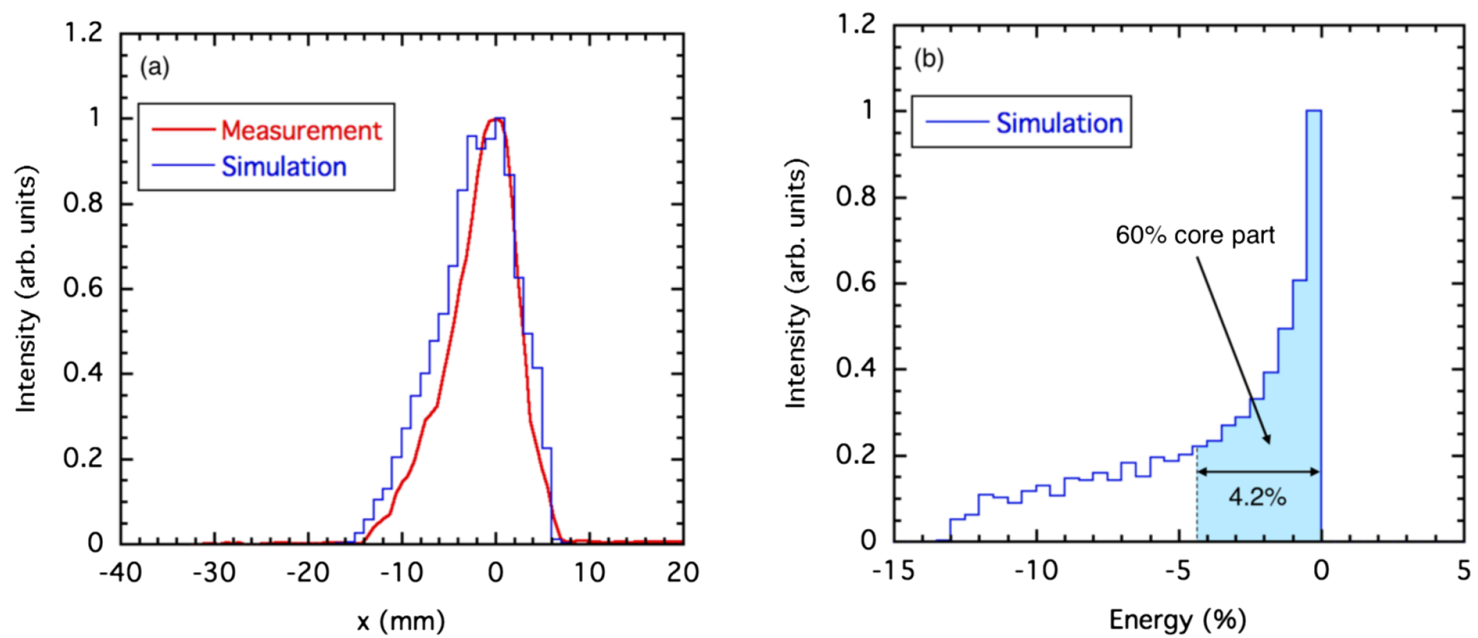

FIG. 11. (a) Measured (red line) and simulated (blue line) beam profiles at energy dispersive section downstream the 238-MHz rf cavity. (b) Energy profile at energy dispersive section downstream the 238-MHz rf cavity obtained with PARMELA simulation.
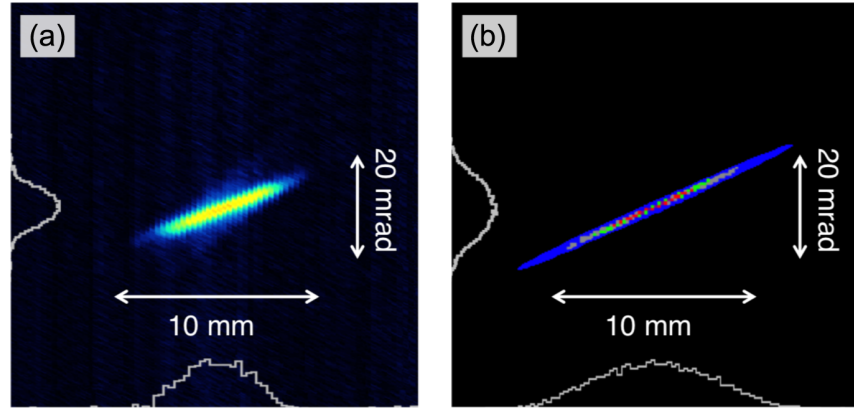

FIG. 12. Phase-space profile of the $500 \mathrm{keV}, 1 \mathrm{nC}$ beam. (a) Measured and (b) simulated phase-space profiles after the rf electron gun system using CST and PARMELA codes. The horizontal and vertical scales are the same in the two figures.

horizontal phase-space profile measured at a scan step of $250 \mu \mathrm{m}$. A normalized horizontal emittance is evaluated to be $4.3 \mathrm{~mm} \mathrm{mrad}$ in $\mathrm{rms}$ for a $90 \%$ core part of the whole electrons by subtracting the background noise from the image data [10]. The normalized emittance decreases to $1.7 \mathrm{~mm} \mathrm{mrad}$ in $\mathrm{rms}$ for a $60 \%$ core part of the whole electrons by cutting an additional $30 \%$ of the electrons with relatively large transverse momenta. Figure 12(b) shows the simulated horizontal phase-space profile, providing normalized emittances of 3.3 and $2.0 \mathrm{~mm}$ mrad in rms for the whole electrons and the $60 \%$ core part, respectively, at the

TABLE III. Measured beam parameters at the exit of the electron gun system.

\begin{tabular}{lc}
\hline \hline Charge & $1 \mathrm{nC}$ \\
Energy & $500 \mathrm{keV}$ \\
Energy spread & $4.2 \%$ (full width of 60\% core part) \\
Normalized emittance & $4.3 \mathrm{~mm} \mathrm{mrad} \mathrm{(rms,90 \%} \mathrm{electrons)}$ \\
Normalized emittance & $1.7 \mathrm{~mm} \mathrm{mrad} \mathrm{(rms,60 \%} \mathrm{electrons)}$ \\
\hline \hline
\end{tabular}

same location with the same beam parameters as the measurement. We found the observed beam distribution and projected emittance values to be consistent with those of the simulation. Table III summarizes the measured beam performance at the gun exit.

\section{SUMMARY}

We developed a low-emittance rf electron gun using a commercially available gridded thermionic cathode. We made our rf gun simple, compact, and easy to maintain without employing a high-power laser system nor a high voltage pulse modulator system. Our proof-of-performance experiments agreed well with CST and PARMELA simulations: a normalized emittance of $1.7 \mathrm{~mm}$ mrad in rms for the $60 \%$ core part of the whole electrons at a beam energy of $500 \mathrm{keV}$ and a bunch charge of $1 \mathrm{nC}$. Since the projected normalized emittance can be reduced to a sufficiently small level of less than $2.0 \mathrm{~mm}$ mrad by collimating the beam while keeping the bunch charge more than $0.5 \mathrm{nC}$, our $\mathrm{rf}$ gun system is a practical electron source for SXFEL systems.

\section{ACKNOWLEDGMENTS}

The authors thank the staff members of SACLA for their continued support, especially Dr. T. Ishikawa, who allowed us to pursue this development project. We also thank Dr. K. Togawa for useful discussions on emittance evaluations using image data obtained by the slit scan method. In addition, author T. A. also thanks the following people for their practical engineering work and for manufacturing the components with excellent quality: Mr. N. Tendo and Mr. N. Kuwabara of Toyama Co., Ltd.; Mr. T. Sugano, Dr. N. Sigeoka, and Mr. K. Higa of Mitsubishi Heavy Industries Ltd.; Mr. S. Aizawa, Mr. T. Okuyama, Mr. N. Kumazawa, and Mr. S. Fukuoka of Nihon Koshuha 
Co., Ltd.; and Mr. M. Kitamura and Mr. K. Kurogi of Hitachi Zosen Corporation. We gratefully acknowledge all staff members of SPring-8 Service Co., Ltd for their engineering and operation support. The SiC-MOSFETs and $\mathrm{SiC}-\mathrm{SBDs}$ used in the $50-\mathrm{kV}$ Marx generator were developed and supplied by Tsukuba Power Electronics Constellations (TPEC) organized by National Institute of Advanced Industrial Science and Technology (AIST).

[1] P. Emma et al., First lasing and operation of an angstromwavelength free-electron laser, Nat. Photonics 4, 641 (2010).

[2] T. Ishikawa et al., A compact x-ray free-electron laser emitting in the sub-ångström region, Nat. Photonics 6, 540 (2012).

[3] H. Weise et al., Commissioning and lasing of the European XFEL, in Proceedings of the 38th International Free Electron Laser Conference, Santa Fe, NM, 2017 (JACoW, Santa Fe, 2017), p. 9.

[4] C. J. Milne et al., SwissFEL: The Swiss X-ray free electron laser, Appl. Sci. 7, 720 (2017).

[5] I. S. Ko et al., Construction and commissioning of PALXFEL facility, Appl. Sci. 7, 479 (2017).

[6] W. Ackermann et al., Operation of a free-electron laser from the extreme ultraviolet to the water window, Nat. Photonics 1, 336 (2007).

[7] E. Allaria et al., The FERMI free-electron laser, J. Synchrotron Radiat. 22, 485 (2015).

[8] Z. Zhao et al., SXFEL: A soft x-ray free electron laser in China, Synchrotron Radiat. News 30, 29 (2017).

[9] X. J. Wang et al., Design and construction a full copper photocathode rf gun, in Proceedings of the 1993 Particle Accelerator Conference, Washington, DC, 1993 (JACoW, Washington, DC, 1993), p. 3000.

[10] R. Akre et al., Commissioning the linac coherent light source injector, Phys. Rev. ST Accel. Beams 11, 030703 (2008).

[11] M. Krasilnikov et al., Experimentally minimized beam emittance from an L-band photoinjector, Phys. Rev. ST Accel. Beams 15, 100701 (2012).
[12] M. Ferrario and T. Shintake, High performance electron injectors, Rev. Accel. Sci. Techol. 03, 221 (2010).

[13] K. Togawa, T. Shintake, T. Inagaki, K. Onoe, T. Tanaka, H. Baba, and $\mathrm{H}$. Matsumoto, $\mathrm{CeB}_{6}$ electron gun for lowemittance injector, Phys. Rev. ST Accel. Beams 10, 020703 (2007).

[14] T. Asaka et al., Low-emittance thermionic-gun-based injector for a compact free-electron laser, Phys. Rev. Accel. Beams 20, 080702 (2017).

[15] N. Nishimori, T. Watanabe, and H. Tanaka, A highly brilliant compact $3 \mathrm{GeV}$ light source project in Japan, in Proceedings of the 2019 International Particle Accelerator Conference, Melbourne, Australia, 2019 (JACoW, CERN, Geneva, Switzerland, 2019), p. 1478.

[16] C. Bourat, Beam dynamics for FEL injection of the linac preinjector of ELETTRA, Nucl. Instrum. Methods Phys. Res., Sect. A 304, 317 (1991).

[17] P. Sprangle, J. Peñano, B. Hafizi, D. Gordon, S. Gold, A. Ting, and C. Mitchell, High average current electron guns for high-power free electron lasers, Phys. Rev. ST Accel. Beams 14, 020702 (2011).

[18] M. Yamada et al., Beam simulations for a mesh gridded electron gun, in Proceedings of the 23rd Linear Accelerator Meeting in Japan (Tsukuba, 1998), p. 148, in Japanese, https://www.pasj.jp/web_publish/lam1998/F16p12.pdf.

[19] T. Asaka et al. (in preparation).

[20] https://www.cpii.com/product.cfm/9/22/131.

[21] B. E. Carlsten, New photoelectric injector design for the Los Alamos National Laboratory XUV FEL accelerator, Nucl. Instrum. Methods Phys. Res., Sect. A 285, 313 (1989).

[22] C. Kondo et al., Development of $50 \mathrm{kV}$ pulse generator using high voltage SiC-MOSFET for electron gun, in Proceedings of 16th Annual Meeting of Particle Accelerator Society of Japan (Kyoto, 2019), p. 679, in Japanese, https://www.pasj.jp/web_publish/pasj2019/proceedings/ PDF/THPH/THPH031.pdf.

[23] H. Kitai et al., Low on-resistance and fast switching of 13-kV SiC MOSFETs with optimized junction field-effect transistor region, in Proceedings of 29th International Symposium on Power Semiconductor Devices and IC's (Sapporo, 2017), p. 343, https://ieeexplore.ieee.org/ document/7988982. 